\title{
Benign Broncho-esophageal Fistula Presenting as Unresolved Pneumonia
}

\author{
Waseem M. Hajjar, Lamyaa A. Althawadi, Nada A. Bin Semaih, Adnan W. Hajjar and Sami A. Al-Nassar \\ Department of Surgery, College of Medicine, King Saud University, Riyadh, Saudi Arabia
}

\begin{abstract}
Benign acquired broncho-esophageal fistula (BEF) in adults is a very rare entity and has not been reported properly in the literature, compared to malignant BEF. Nonetheless, infection has predisposed most of the reported benign acquired BEF cases. We report here a case of tuberculous BEF, in a patient with a history of pulmonary tuberculosis (TB). He presented with recurrent chest infections, and choking. Upper gastrointestinal (GI) endoscopy showed BEF due to tuberculous mediastinal lymphadenopathy, and the patient was managed by surgery successfully. He was prepared for surgery for one month by nutritional support and anti-tuberculous treatment.
\end{abstract}

Key Words: Broncho-esophageal fistula, Mycobacterium tuberculosis, Surgery.

How to cite this article: Hajjar WM, Althawadi LA, Semith NAB, Hajjar AW, Al-Nassar SA. Benign broncho-esophageal fistula present as unresolved pneumonia. J Coll Physicians Surg Pak 2019; 29 (Supplement 2):S148-S150.

\section{INTRODUCTION}

Broncho-esophageal fistula (BEF) is rarely seen in adults, and is mostly caused by malignancy. ${ }^{1}$ However, it might be produced by benign conditions such as trauma or infection. ${ }^{1}$ Chest infection caused by mycobacterium tuberculosis (TB) is common, especially in endemic countries. However, formation of BEF as a complication of TB infection is significantly rare. ${ }^{2}$

In addition, due to vague unspecific presentation of BEF patients, they are usually misdiagnosed, or the diagnosis may be delayed; and consequently, the morbidity and mortality are increased. ${ }^{1}$ Diagnosis of BEF is made usually by endoscopy or contrast studies. Surgical management of BEF is the treatment of choice ${ }^{1}$, although a minority of patients are not suitable for immediate surgical intervention. ${ }^{3}$

As revealed in the literature, BEF formation in TB patients is caused by granulomatous inflammatory processes and the caseating necrosis of mediastinal lymph nodes, which eventually erode and rupture into the wall of esophagus and bronchus during this active infection. 4,5

\section{CASE REPORT}

A 47-year male patient, known case of adrenocortical insufficiency on replacement therapy of steroids (prednisolone), presented to the outpatient department of our hospital complaining of productive cough, inter-

Correspondence to: Dr. Waseem M. Hajjar, Department of Surgery, College of Medicine, King Saud University, King Khalid University Hospital, Riyadh, P.O. Box 7805, Saudi Arabia

E-mail:washajjar@yahoo.com

Received: March 25, 2019; Revised: July 26, 2019;

Accepted: August 06, 2019 mittent fever for some time, along with nausea and vomiting. He lost $11 \mathrm{Kgs}$ of weight in the last 12 months, experienced night sweats, and anorexia. Patient has had recurrent pneumonia, which was mainly affecting the left lower lobe. Detailed history revealed that the patient had experienced choking-like symptoms and coughing on ingestion of liquids.

He had history of pulmonary TB infection 8 years prior to presentation, with mediastinal lymph nodes involvement. He was treated for two years with anti-TB medications.

General examination, chest examination and other systemic examinations were unremarkable.

All his blood investigations were within normal range. Culture of three sputum samples for acid-fast bacilli (AFB) was negative. Electrocardiogram and echocardiogram were also unremarkable. Definitive diagnosis of the underlying cause was not very clear, due to unremarkable results. Hence, esophageal pathology was suspected from the history of the patient. The upper gastrointestinal (GI) tract endoscopy revealed mild nonspecific esophagitis, and an opening of a narrow fistulous tract at $34 \mathrm{~cm}$ from the incisors between the esophagus and the left lower lobe bronchus (Figure $1 \mathrm{~A}, \mathrm{~B}$ ). In addition, abundant secretions were seen around the fistulous tract opening. Esophageal aspirate of these secretions and the esophageal tissue biopsy around this fistula showed positive Ziehl-Neelsen stain for AFB.

CT-scan chest and contrast study of barium swallow confirmed the above findings of the fistula formation between the esophagus and the left lower lobe bronchus (basal segment). (Figures 2, and $3 \mathrm{~A}$ ).

Patient was started on anti-TB medications, and at the same time. the steroids were tapered down. During this 


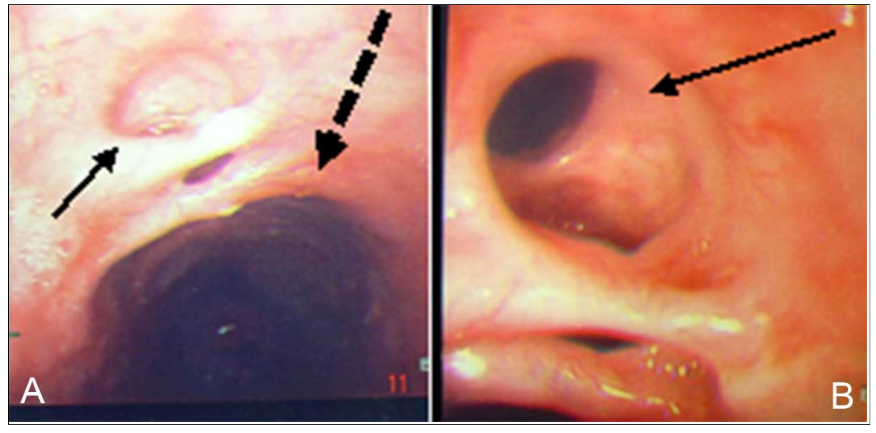

Figure 1 (A, B): Upper GI endoscopy close view showing the fistula between the left lower bronchus (basal segment) and the esophagus. Fistula (straight arrow) esophagus (dash arrow).

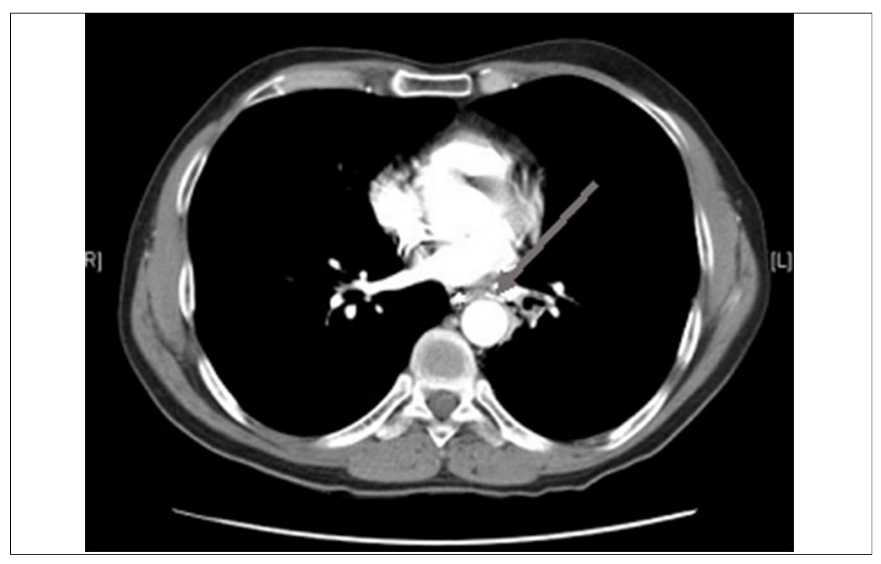

Figure 2: CT scan chest, enhanced with I.V and oral contrast showing fistula formation between the esophagus and the left lower lobe bronchus.

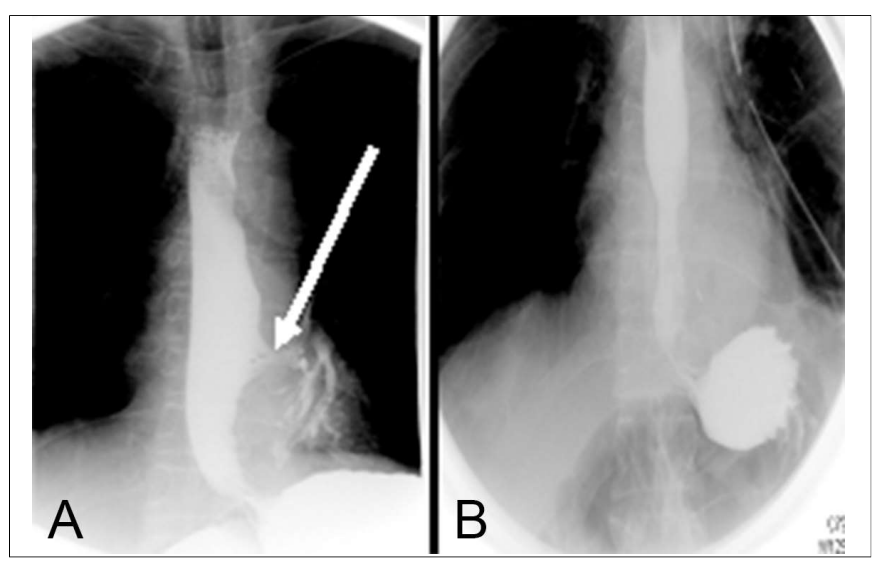

Figure $3(A, B)$ : Barium swallow study showing the fistula between the lower part of the esophagus and the left lower lobe bronchus (A). Barium swallow postoperation shows healed fistula chest tube in position (B).

period, nutritional support was established by nasoenteric feeding and the tube was passed down to the duodenum under $\mathrm{X}$-ray screening. He remained on this management plan for one month, in order to settle the infection, decrease the chances of recurrence, to promote post-surgical wound healing, and ultimately improving the surgical outcome. Later on, he underwent left thoracotomy and exploration. Dissection of the esophagus revealed the fistula formation to the left lower lobe bronchus, with multiple mediastinal lymph nodes, which were resected and sent for histopathology. Closure of the fistula was done by stapler at the bronchial side, and suturing the esophageal side in two layers, and covering it with pericardial fat as a buttress.

Postoperatively, patient was continued on anti-TB medications through the naso-enteric tube. He was also started on total parenteral nutrition (TPN). Three weeks later, barium swallow showed no leakage (Figure 3B). Thus oral diet was resumed.

Histopathology of the lymph nodes showed reactive follicular hyperplasia with granuloma formation. Patient was discharged home in a very good condition; and he remained very well under close follow-up in the surgical and medical outpatient clinic for 8 years.

\section{DISCUSSION}

BEF is a rare entity and mostly caused by malignant processes of the esophagus, or other adjacent anatomical structures. It can be caused by benign conditions such as TB infection or trauma. ${ }^{1} \mathrm{Ahn}$, et al., showed that benign acquired BEF in adults accounted for only $4.8 \%$ (18 of 368) of all BEF cases, while malignant process accounted for $95.2 \%$ (350 of 368) of the cases. ${ }^{1}$ Among benign acquired causes, infection was predominant in $66.6 \%$ (12 of 18); and of these benign BEF cases, TB was identified in all 12 patients, and eight patients developed BEF after they were treated for pulmonary TB.1 Spalding et al., in a retrospective study on 7 patients with acquired benign BEF, demonstrated that $85.7 \%$ (6 out of 7 ) were predisposed by infection and Mycobacterium tuberculosis was the causative agent in $42.8 \%$ (3 out of 7 ) cases. ${ }^{4}$

Our patient had history of pulmonary TB with mediastinal lymph node involvement eight years prior to presentation, and was treated with anti-TB medications for two years. Despite this management and being immune-competent, he developed tuberculous BEF, which was probably due to corticosteroids he was taking for adrenocortical insufficiency.

Association between the use of corticosteroids and the risk of reactivation of TB infection was studied by Jick et al., and they showed that patients exposed to corticosteroids have 5 -fold higher risk of re-infection with TB. ${ }^{6}$

Recurrent chest infections and respiratory symptoms were the presenting features in nearly all benign acquired BEF patients caused by infection. However, some patients also complain of choking with liquid ingestion, which raises the suspicion of esophageal pathology, and helps to establish an early and definitive diagnosis. Thus, the diagnosis of BEF in patients who are complaining of isolated respiratory symptoms and recurrent chest infections is usually delayed.

Diagnosis of BEF is confirmed by upper GI endoscopy, fiber optic bronchoscopy, and barium swallow (Figures $1 \mathrm{~A}, \mathrm{~B}$, 
$3 A)$. Surgical management has proven to be the best option for such patients. However, a minority of patients might not be fit for immediate surgery ${ }^{3}$, like our patient, as he needed nutritional support; and delay in surgery, tapering down his steroids, and starting him on intravenous IV anti-TB medications for one month, in order to settle the infection, decrease the chances of recurrence, to promote post-surgical wound healing, and ultimately improving the surgical outcome.

Benign BEF caused by TB is a very rare entity. However, detailed history, examination and proper investigations help to establish early and definitive diagnosis. Proper management in the form of medical and surgical intervention is warranted to achieve complete cure.

\section{PATIENT'S CONSENT:}

Written informed consent was obtained from the patient, who participated in this study, for publication of this manuscript and any accompanying images.

\section{CONFLICT OF INTEREST:}

Authors declared no conflict of interest.

\section{AUTHORS' CONTRIBUTION:}

All the Authors declared of taking responsibility and contri-buted equally in the literature review and in the cons-truction of the body of the manuscript. Moreover, reviewed the article before submission not only for spelling and grammar but also for its intellectual content.

\section{REFERENCES}

1. Ahn JY, Jung HY, Choi JY, Kim MY, Lee JH, Choi KS, et al. Benign bronchoesophageal fistula in adults: Endoscopic closure as primary treatment. Gut Liver 2010; 4:508-13.

2. Andreu J, Cáceres J, Pallisa E, Martinez-Rodriguez M. Radiological manifestations of pulmonary tuberculosis. Eur $\mathrm{J}$ Radiol 2004; 51:139-49.

3. Mangi AA, Gaissert HA, Wright CD, Allan JS, Wain JC, Grillo HC, et al. Benign broncho-esophageal fistula in the adult. Ann Thorac Surg 2002; 73:911-5.

4. Spalding AR, Burney DP, Richie RE. Acquired benign bronchoesophageal fistulas in the adult. Ann Thorac Surg 1979; 28: 378-83.

5. Andreu J, Cáceres J, Pallisa E, Martinez-Rodriguez M. Radiological manifestations of pulmonary tuberculosis. Eur $J$ Radiol 2004; 51:139-49.

6. Jick SS, Lieberman ES, Rahman MU, Choi HK.Glucocorticoid use, other associated factors, and the risk of tuberculosis. Arthritis Rheum 2006; 55:19-26.

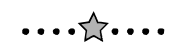

